\title{
Continuous Tongue Suction as a Potential Therapy for Obstructive Sleep Apnea: A Feasibility Study
}

\author{
Tatsuya Fukuda, DDS 1 ; Yoichiro Takei, RPSGT ${ }^{1}$; Hideaki Nakayama, MD, PhD ${ }^{1,2}$; \\ Yuichi Inoue, MD, PhD ${ }^{1,2}$; ${ }^{*}$ Satoru Tsuiki, DDS, PhD ${ }^{1,3,4}$
}

\begin{abstract}
${ }^{1}$ Japan Somnology Center, Institute of Neuropsychiatry, Tokyo, Japan; ${ }^{2}$ Department of Somnology, Tokyo Medical University, Tokyo, Japan; ${ }^{3}$ Aging and Geriatric Dentistry, Faculty of Dentistry, Tohoku University, Sendai, Japan; ${ }^{4}$ Department of Oral Health Sciences, Faculty of Dentistry, The University of British Columbia, Vancouver, Canada
\end{abstract}

\begin{abstract}
During wakefulness, apneic events, even in patients with severe obstructive sleep apnea (OSA), rarely occur regardless of the presence or absence of such episodes while asleep, because the augmented activity of the genioglossus muscle acts to patent the upper airway by maintaining the tongue in position. Hence, it is reasonable to hypothesize that OSA could be alleviated if the awake tongue position is maintained despite a sleep-related reduction in genioglossus muscle activity. The median (interquartile range) respiratory event index was significantly reduced with continuous tongue suction (23 [16-27] to 8 [7-14] events/h, $\mathrm{P}=0.043)$ in 5 patients who successfully completed the protocol. Because this approach does not require either positive airway pressure or mandibular advancement, it makes it possible to completely avoid the adverse effects associated with the use of nasal continuous positive airway pressure and/or mandibular advancement devices in patients for whom nasal continuous positive airway pressure and mandibular advancement devices are contraindicated.
\end{abstract}

Keywords: Obstructive sleep apnea; Oral appliance; tongue; alternative therapy

Citation: Fukuda T, Takei Y, Nakayama H, Inoue Y, Tsuiki S. Continuous tongue suction as a potential therapy for obstructive sleep apnea: A feasibility study. J Dent Sleep Med. 2020;7(3)

\section{INTRODUCTION}

During wakefulness, apneic events, even in patients with severe obstructive sleep apnea (OSA), hardly occur regardless of the presence or absence of such episodes while asleep, because the augmented activity of the genioglossus muscle helps maintain a patent upper airway by keeping the tongue in the awake position. ${ }^{1}$ Hence, OSA could be alleviated if the awake tongue position is maintained despite the sleep-related reduction in genioglossus muscle activity, which represents a different approach to the treatment of OSA than the use of nasal continuous positive airway pressure (nCPAP) or mandibular advancement devices. ${ }^{2}$ Exploratory results are discussed to support the hypothesis that the concept of maintaining the awake tongue position by continuous tongue suction per se may be applicable as a potential treatment for OSA. Part of the current study has been reported previously in a case report. ${ }^{2}$

\section{REPORT OF CASE}

In all of the eligible patients with OSA in this study, treatment with mandibular advancement devices was contraindicated because of dental and/or temporomandibular joint problems. This group included patients in whom nCPAP and tongue-stabilizing devices were unsuccessful. ${ }^{3}$ These patients were consecutively contacted and invited to participate in the current study between March 2010 and June 2012. A total of seven patients agreed to participate and provided their written informed consent after the aims, detailed methods, benefits, and potential risks were fully explained by the principal investigator (ST). "Potential risks of the tongue position controller (TPC) included soreness and/or discomfort on the tongue surface, excessive saliva, discomfort of the lower teeth and gums, noise from the pressure controller, and discomfort of the lips". ${ }^{2}$ The protocol of this study was approved by the Ethics Committee of the Institute of Neuropsychiatry, Tokyo (approval no. 43) and was conducted in accordance with the amended Declaration of Helsinki.

The details of a tongue position controller that entails a tongue positioner, a suction tube, and a pressure controller for continuous tongue suction, have been described previously 2,4 (Figure 1). "The custom-made tongue positioner (Figure 1, C through $\mathrm{F}$ ) is fabricated on plaster dental casts of the mandibular dental arch. It is made of polyolefin and is designed to encircle approximately one-half to two-thirds of the tongue surface by a dome-type capsule and a lower dental splint. The capsule and the part of the lower splint that directly touches the tongue form two layers. The outer layer of the capsule smoothly extends from the lingual surface of the lower splint. The inner layer, with small holes (2 to $3 \mathrm{~mm}$ in diameter), directly touches the tongue surface (Figure 1, E). The border of the inner and outer layers is firmly closed 
with polyolefin. Thus, the two layers form a closed space $2 \mathrm{~mm}$ thick. Negative pressure is applied from a pressure generator (0 to $-4 \mathrm{~cm} \mathrm{H}_{2} \mathrm{O}$, Seastar Corporation Inc. Ltd., Tokyo, Japan) via a catheter made of polyethylene (diameter $3 \mathrm{~mm}$, Seastar Corporation Inc. Ltd., Tokyo, Japan) that is connected to a hole (diameter 3 to $5 \mathrm{~mm}$ ) located at the ventral part of the outer layer of the capsule. Accordingly, when negative pressure is applied from the pressure generator, the air in the closed space is sucked through the small holes in the inner layer of the capsule, which creates suction over the surface of the tongue. This design for a tongue positioner has the advantage of minimizing pain and/or discomfort on the tongue surface because suction is applied through the small holes that are located throughout the inner layer of the capsule, rather than directly through the hole that is connected to the tip of the catheter. The pressure generator sucks the air with a manually adjusted flow rate of 100 to $3,000 \mathrm{~mL} / \mathrm{min}$. The lateral pressure in the catheter is measured with a custommade manometer and is expressed as $\mathrm{cm} \mathrm{H}_{2} \mathrm{O}$ ". ${ }^{2}$

Figure 1. A, Patient with a tongue position controller in place. B, The device allows jaw opening. $\mathbf{C}$ through $\mathbf{F}$, Various views of the tongue position controller. C, Anterior. D, Lateral. E, Posterior. F, Posterior oblique.
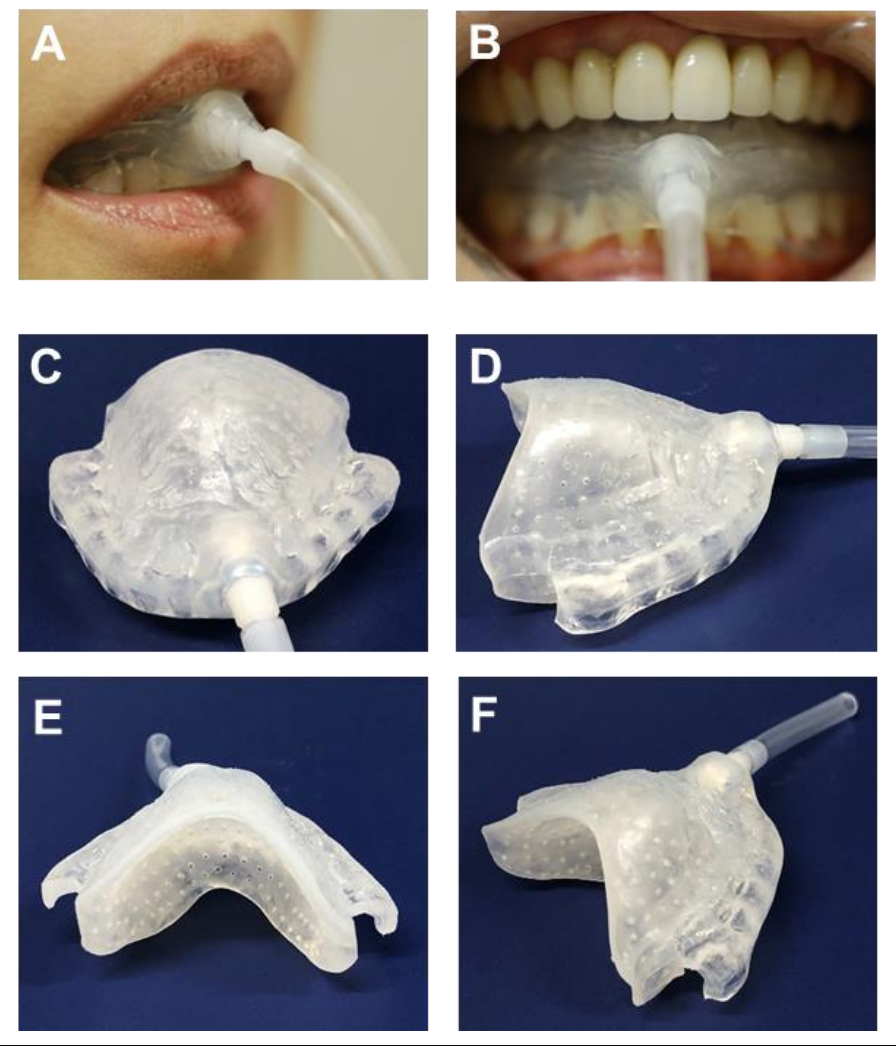

A six-channel portable monitor (PMP-300E, Pacific Medico Co., Ltd., Japan) was used to manually assess the baseline respiratory event index (REI) according to American Academy of Sleep Medicine standards (version 2.1 ) by a registered polysomnographic technologist
(YT). 5,6 "Initially, each patient was instructed to use the tongue position controller with a negative pressure of $-4 \mathrm{~cm}$ $\mathrm{H}_{2} \mathrm{O}$. When patients complained of dry mouth and mild soreness of the tongue the morning after the first night, the negative pressure load was slightly reduced (by approximately -0.5 to $-1 \mathrm{~cm} \mathrm{H} 2 \mathrm{O}$ ) the next night. This process was repeated during a 1-month acclimatization period until the maximum comfortable limit of negative pressure was achieved, at which patients thought that the tongue position was properly maintained without any discomfort. It was considered that the negative pressure was sufficiently titrated when a cessation of snoring/apnea was reported by a bed partner and/or subjective daytime sleepiness was resolved". ${ }^{2}$ A follow-up home monitoring test was performed after the subject was acclimated to the tongue position controller to determine REI with continuous tongue suction. All statistical analyses were performed in a blind manner with respect to the subject's background information. Data were presented as median values (interquartile range) and analyzed on both an intention-to-treat basis and a per-protocol basis. ${ }^{7}$ Wilcoxon signed rank tests were used to compare the differences between the baseline and follow-up values. A value of $P<0.05$ was considered to indicate statistical significance.

During the trial, one of the seven patients (YS) dropped out of the study because of tongue discomfort. None of the remaining six patients reported problems with swallowing during sleep, significant discomfort or pain of the tongue, teeth, gums or temporomandibular joint. Dry mouth $(n=2)$, noise due to the accumulation of saliva in the suction tube $(n=1)$, difficult adjustment of suction pressure $(n=3)$, difficult device maintenance $(n=2)$, and disturbed lateral sleep position due to a suction tube $(n=3)$ were reported. One subject (HM) reported that the TPC fell off during the follow-up home monitoring test. Consequently, six of the original seven patients completed both the baseline and follow-up evaluations, and five of the seven subjects successfully completed the protocol.

Figure 2 shows an example montage of the home monitoring test and Table 1 as well as Figure 3 shows the effects of continuous tongue suction on REI. In 6 patients, excluding the patient who withdrew, the REI was changed from 20 (12-26) to 11 (7-15) events/h ( $\mathrm{P}=0.173)$. The REI was significantly reduced with continuous tongue suction (23 [16-27] to 8 [7-14] events/h, $\mathrm{P}=0.043$ ) in 5 patients when we further excluded the patient (HM) whose TPC fell off during the study.

\section{DISCUSSION}

The maintenance of tongue position by continuous tongue suction improved REI in patients with OSA. Because this approach does not require either positive airway pressure or mandibular/tongue advancement, it makes it possible to completely avoid the adverse effects 
Figure 2. An example montage of home monitoring at baseline (left) and under continuous tongue suction as a follow-up (right).

Baseline

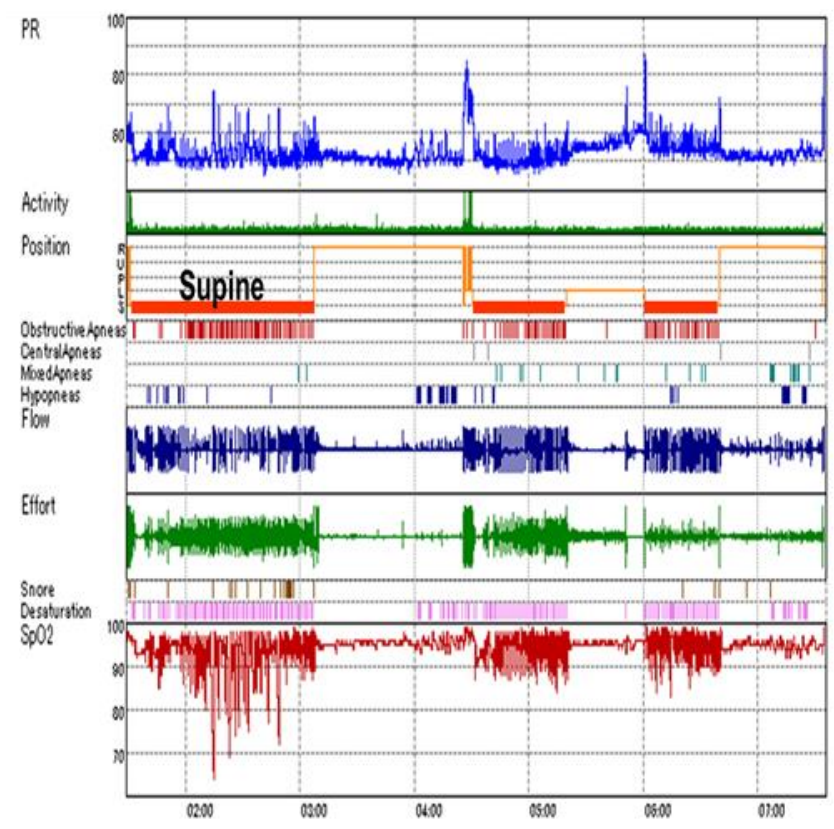

\section{Tongue position controller}

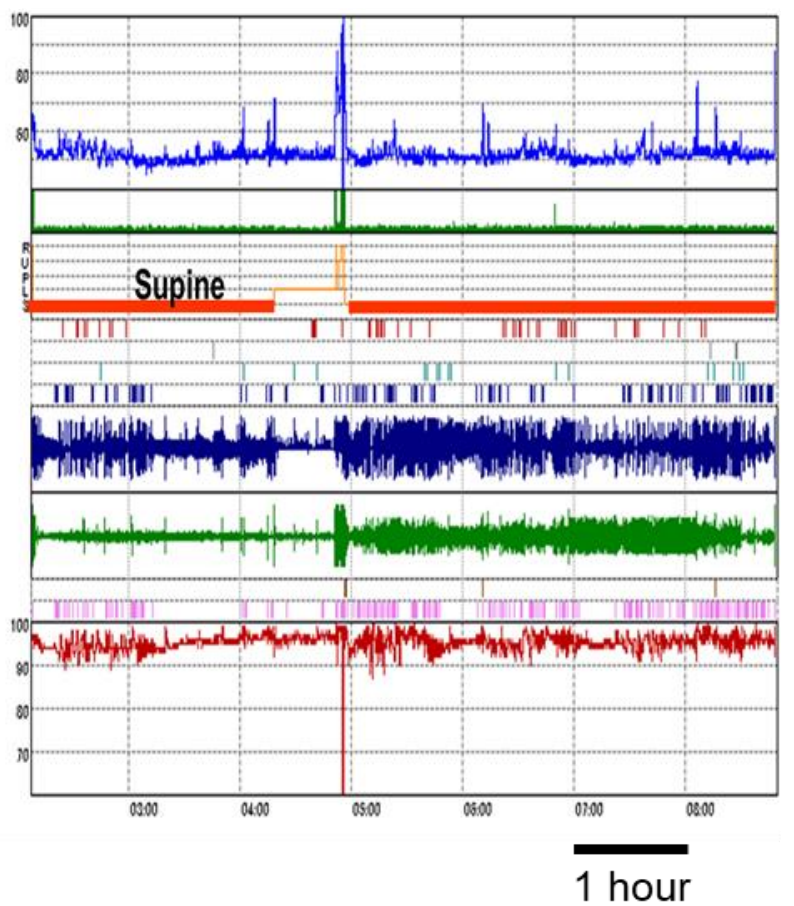

The lowest percutaneous oxygen desaturation (bottom) increased from $64 \%$ to $87 \%$ (patient 1, KM, 71 year-old man with obstructive sleep apnea with compromised dentitions who could not tolerate either nasal continuous positive airway pressure or a tongue-stabilizing device). Note that this favorable change is likely independent of body position.

Figure 3. Respiratory event index (REI) and nadir $\mathrm{SpO}_{2}$ at baseline and with tongue position controller in place. There was no significant change in body mass index during the study. Note that the device of patient 5 fell off during the test night.
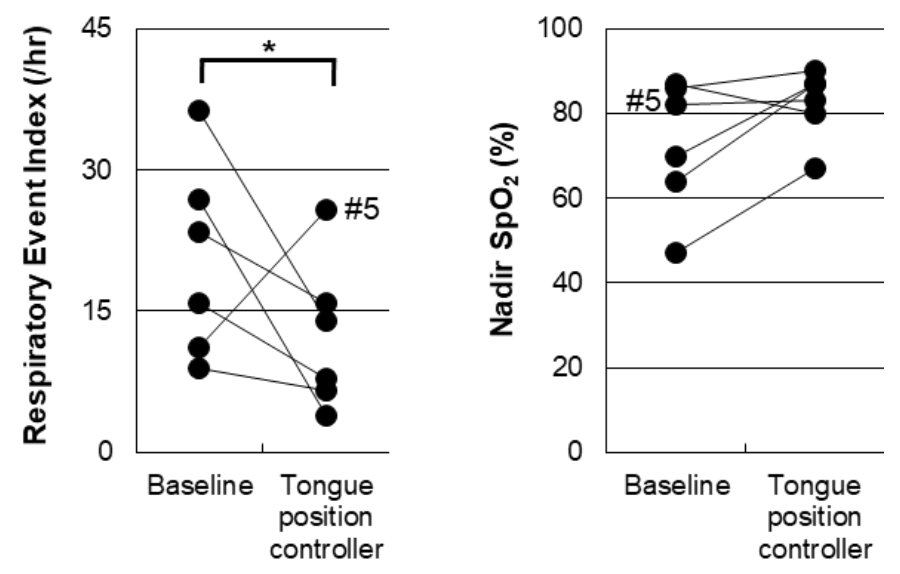

associated with the use of nCPAP and/or oral appliances and can be used in patients in whom nCPAP and mandibular/tongue advancement devices are contraindicated. "For example, because the mandible was not advanced at all because the tongue positioner of the tongue position controller was merely applied to the lower dentition, temporomandibular joint side effects and proclination as well as retroclination of incisors could be avoided. $^{2}$ It could be stated that the idea of the TPC being similar to that of a tongue-retaining device is that it directly approaches to the tongue. However, the tongue retaining device does not entail methods when the suction pressure is incomplete. The TPC can overcome such deficits of the tongue-retaining device because the TPC incorporates a closed-loop system where the tongue is held by active and continuous suction. Furthermore, the discomfort often caused by tongue protrusion with the tongue-retaining device can be reduced with the TPC because the TPC maintains the normal tongue position (i.e., no tongue protrusion), which could lead to better adherence". ${ }^{2}$

Conversely, the fact that two patients did not satisfactorily tolerate the TPC implies that the device needs to be modified, although we speculate that the dropouts in 
Table 1. Effects of continuous tongue suction on respiratory event index.

\begin{tabular}{|c|c|c|c|c|c|c|c|c|}
\hline \multirow[t]{2}{*}{ Initials } & \multirow[t]{2}{*}{ Patient No. } & \multirow[t]{2}{*}{ Sex } & \multirow[t]{2}{*}{ Age } & \multirow[t]{2}{*}{ BMI } & \multicolumn{2}{|c|}{ REI (events/h) } & \multicolumn{2}{|c|}{ Nadir $\mathrm{SpO}_{2}(\%)$} \\
\hline & & & & & Baseline & TPC & Baseline & TPC \\
\hline $\mathrm{KM}$ & 1 & M & 71 & 25 & 23 & 16 & 64 & 87 \\
\hline JT & 2 & $\mathrm{~F}$ & 54 & 19 & 27 & 4 & 87 & 80 \\
\hline NM & 3 & M & 66 & 26 & 36 & 14 & 47 & 67 \\
\hline $\mathrm{KN}$ & 4 & $\mathrm{M}$ & 27 & 26 & 16 & 8 & 70 & 87 \\
\hline $\mathrm{HM}$ & 5 & $\mathrm{M}$ & 39 & 20 & 11 & 26 & 82 & 83 \\
\hline SA & 6 & $\mathrm{~F}$ & 65 & 22 & 9 & 7 & 86 & 90 \\
\hline & $\mathrm{N}=6$ & & $60(43-66)$ & $24(20-26)$ & $20(12-26)$ & $11(7-15)$ & $76(66-85)$ & $85(84-90)$ \\
\hline & (5 excluded) & & $65(54-66)$ & $25(22-26)$ & $23(16-27)$ & $8(7-14)^{a}$ & 70 (64-86) & $87(80-87)$ \\
\hline
\end{tabular}

$\mathrm{BMI}$, body mass index; $\mathrm{REI}$, respiratory event index; $\mathrm{SpO}_{2}$, percutaneous oxygen saturation; TPC, tongue position controller.

a $\mathrm{P}<0.05$ versus baseline. Data are presented as median values (interquartile range) and analyzed on both an intention-to-treat basis $(\mathrm{N}=6)$ and a per protocol basis $(\mathrm{N}=5)$.

this study does not necessarily undermine the concept of maintaining the awake tongue position. Rather, whether continuous tongue suction is feasible for OSA treatment or whether the current study is an example of a type I error should be evaluated in future trials after renovation and/or upgrade of the device.

\section{ACKNOWLEDGEMENTS}

The current study was supported in part by the Grantsin-Aid for Scientific Research, Japan Society for the Promotion of Science KAKENHI (Grant numbers 17K11793, 19K10236, and 19K10437). This is not an industry-supported study and the TPC is registered with the Japan Patent Office. The authors are grateful to Eri Kikuta and Yusaku Soga (Aso International Inc. Ltd, Tokyo Japan) for their expert technical assistance. We also thank Akihito Eguchi, ME (TMI Associates), Dr. Shiroh Isono, $\mathrm{MD}, \mathrm{PhD}$, and Dr. Tatsuo Kagimura, $\mathrm{PhD}, \mathrm{MPH}$ for their critical comments on the design of TPC and the data analyses.

\section{REFERENCES}

1. White DP. Pathogenesis of obstructive and central sleep apnea. Am J Respir Crit Care Med 2005;172(11):1363-1370.
2. Tsuiki S, Isono S, Minamino O, et al. Tongue position controller as an alternative treatment for obstructive sleep apnea. Sleep Breath 2012;16(4):957-960.

3. Yanagihara M, Tsuiki S, Setoguchi Y, Inoue Y. Treatment of obstructive sleep apnea with a tongue-stabilizing device at a single multidisciplinary sleep center. J Dent Sleep Med 2016;3(2):43-47.

4. Tsuiki S, Minamino O. Tongue position controller. United States Patent Application Publication; US 2011/0259346 A1. Publication date; October 27, 2011.

5. Berry RB, Brooks R, Gamaldo CE, et al, for the American Academy of Sleep Medicine. The AASM Manual for the Scoring and Associated Events: Rules, Terminology and Technical Specifications, Version 2.1. Darien, IL: American Academy of Sleep Medicine; 2014.

6. Kushida CA, Cardell C, Black S, Khouzam A. Comparison of a new type 3 portable monitor for OSA detection vs. in-lab polysomnography [abstract]. Sleep 2009;32:A385.

7. Galløe AM, Rasmussen HS, Jørgensen LN, et al. Influence of oral magnesium supplementation on cardiac events among survivors of an acute myocardial infarction. BMJ 1993;307(6904):585-587.

\section{SUBMISSION \& CORRESPONDENCE INFORMATION}

Submitted for publication July 16, 2019

Submitted in final revised form January 14, 2020

Accepted for publication February 3, 2020

Address correspondence to: Satoru Tsuiki, DDS, PhD, Division of Dental Sleep Medicine, Japan Somnology 
Center, Institute of Neuropsychiatry 5-10-10, Yoyogi, Shibuya-ku Tokyo, Japan 151-0053; Tel: +81-3-68048995; $\quad$ Fax: +81-3-6804-8996; E-mail: tsuiki@somnology.com

\section{DISCLOSURE STATEMENT}

This work was performed at the Institute of Neuropsychiatry, Tokyo, Japan. All authors have seen and approved the final version of this manuscript. This report was supported in part by a Grant-in-Aid for Scientific Research KAKENHI (Grant Numbers 17K11793 for TF, $19 \mathrm{~K} 10236$ for ST, and $19 \mathrm{~K} 10437$ for YT) from the Japanese Society for the Promotion of Science. This is not an industry-supported study and the tongue position controller is registered with the Japan Patent Office. Written informed consent was obtained from the patient regarding the anonymous use of the patient's data for presentation and/or publication. The authors have indicated no conflicts of interest. 